\title{
EPIDEMIOLOGICAL PROFILE OF PATIENTS WITH RHEUMATOID ARTHRITIS IN AN AMBULATORY IN
} FEIRA DE SANTANA-BA

Filipe Mota Freitas (Universidade Estadual de Feira de Santana, Feira de Santana, BA, Brasil), João Mário Aguiar Abrantes Dourado (Universidade Estadual de Feira de Santana, Feira de Santana, BA, Brasil), Ana Teresa Amoedo (Universidade Estadual de Feira de Santana, Feira de Santana, BA, Brasil), Blenda Maria dos Santos Erdes (Universidade Estadual de Feira de Santana, Feira de Santana, BA, Brasil), Dyalle Costa e Silva (Universidade Estadual de Feira de Santana, Feira de Santana, BA, Brasil), Evelly Cerqueira de Amorim Paixão (Universidade Estadual de Feira de Santana, Feira de Santana, BA, Brasil), Jordyr Matheus Magalhães Rocha (Universidade Estadual de Feira de Santana, Feira de Santana, BA, Brasil), Laura Sabrina de Almeida Fernandes (Universidade Estadual de Feira de Santana, Feira de

Santana, BA, Brasil), Lucas dos Santos Silva (Universidade Estadual de Feira de Santana, Feira de Santana, BA, Brasil), Michele Karen Pereira Clementino (Universidade Estadual de Feira de Santana, Feira de Santana, BA, Brasil), Rosimary Alves da Silva (Universidade Estadual de Feira de Santana, Feira de Santana, BA, Brasil)

\section{BACKGROUND}

Rheumatoid arthritis (RA) is a chronic inflammatory disease that can affect several joints. The cause is unknown and affects women twice as often as men. It usually starts between 30 and 40 years and its incidence increases with age. The most common symptoms are pain, swelling, heat and redness in any joint of the body especially hands and wrists. The inflamed joints provoke morning stiffness, fatigue and with the progression of the disease, there is destruction of the articular cartilage and the patients can develop deformities, being more common in peripheral joints like the swan neck fingers, button fingers, ulnar deviation and hallux valgus. The objective of this study was to analyze the epidemiological profile of patients with rheumatoid arthritis in an outpatient clinic in Feira de Santana-BA.

\section{MATERIALS AND METHODS}

This is an epidemiological, descriptive, quantitative and retrospective study carried out in an outpatient clinic in the interior of Bahia. The sample consisted of the analysis of 366 medical records from February 2008 to May 2019, being excluded from the study the patients who were discharged from the clinic or who did not have a diagnosis of rheumatic disease. In this study, a form was used to collect the data of patients with rheumatoid arthritis in which the following variables were analyzed: sex, age, ethnicity, life habits and therapeutic drugs. Then the collected data were inserted into an electronic database and analyzed using the statistical program Epidata Analysis V2.2.3.187.

\section{RESULTS}

Of the 366 records analyzed, $37.4 \%$ (137) of the patients with the diagnosis of rheumatoid arthritis were found, $84.7 \%$ (116) of the female sex, $13.1 \%$ (18) $2 \%$ (3) had no identified sex. The age ranged from 13 to 81 years, with a mean age of 52 years. As for ethnicity, 38.7\% (53) mulattoes, $26.3 \%$ (36) blacks, $19.7 \%$ (27) whites and $15.3 \%$ (21) did not have ethnicity identified. In relation to life habits, $19.7 \%$ (27) stated that they were smokers, $16.8 \%$ (23) were alcoholics and $15.3 \%$ (21) practiced physical activity. As for therapy, $70 \%$ (96) of the patients used synthetic DMARD, 10.2\% (13) used biological DMARD and 27\% used Hydroxychloroquine.

\section{CONCLUSION}

Rheumatoid arthritis is a disease that can cause motor limitations in affected patients, therefore, it is necessary that the diagnosis be made quickly so that the treatment is more effective and the patient develops the minimum of complications. 